\title{
Morfologia Externa de Triatoma ryckmani Zeledón \& Ponce, 1972 Vista Através da Microscopia Eletrônica de Varredura
}

\author{
Rodolfo U Carcavallo, Rodrigo Zeledón*, José Jurberg**/+, \\ Itamar Galindez $* * *$
}

\begin{abstract}
CIBION/COMICET, Serrano 669 (1414) Buenos Aires, Argentina *Escuela Medicina Veterinária, Universidad Nacional de Heredia, Apdo Postal 1466, 2050 San Jose, Costa Rica **Laboratório Nacional e Internacional de Referência em Taxonomia de Triatomíneos, Departamento de Entomologia, Instituto Oswaldo Cruz, Av. Brasil 4365, 21045-900 Rio de Janeiro, RJ, Brasil ***Universidad de Los Andes, Laboratório de

Biología de Triatominae, Trujillo, Venezuela
\end{abstract}

External Morphology of Triatoma rickmani Zeledón \& Ponce, 1972 by Scanning Electron Microscopy - A male of Triatoma ryckmani Zeledón \& Ponce, 1972, was studied by scanning electron microscopy. Only few specimens of this species are known. In this paper, some structures from the head, thorax, abdomen and distal region of the second leg are shown. Some of them could have taxonomic importance, as the oculo-ocellar region, the buccula, the anterolateral angle of the collar, the scutellum with the process longer than the main body, the stridulatory sulcus with an unusual backward vermiform area, and the tibia-tarsal articulation, with a spongy fossula. The last structure was absent in specimens previously studied (Lent \& Wygodzinsky 1979). Differences between this specimen and others previously described by several authors are dicussed.

Key words: Triatoma ryckmani - morphology - taxonomy

Triatoma ryckmani foi descrita em 1972, por Zeledón e Ponce, de um exemplar fêmea procedente de Honduras. Lent \& Wygodzinsky (1979) redescrevem a espécie analisando quatro exemplares fêmeas, uma delas o tipo, outra procedente também de Honduras e duas da Guatemala. Os três últimos exemplares foram encontrados em uma carga aérea proveniente da América Central no Aeroporto de Miami, Estados Unidos. Os exemplares da Guatemala chegaram ao aeroporto em um carregamento de bromélias. Sherlock e Morera (1988) descreveram o alótipo macho capturado sob cascas de uma árvore, Enterolobium cycloarpum, na Costa Rica.

Baseado na descrição desses cinco exemplares, foi firmado um conceito sistemático e taxonômico mais preciso desta rara espécie, de hábitos aparentemente silvestres, apesar de uma fêmea, o holótipo, ter sido encontrada em um acampamento militar onde pernoitavam os soldados.

Realizado com auxílio do CNPq, Convênio BIRD/FNS/ FIOCRUZ, 027/93, Commission European Comunities TS3 CT 920092, Instituto Nacional de Biodiversidad (INBIO), Costa Rica.

+Autor correspondente: Fax: +55-21-290.9339

Recebido em 15 de abril de 1996

Aceito em 2 de julho de 1996

\section{MATERIAIS E MÉTODOS}

Exemplar macho, com os seguintes rótulos: (1) Est. Santa Rosa, 800m, P.N. Guanacaste. Prov. Guan. Costa Rica. PMG. Inventário de Biodiversidad Ene 1990. L.M. 313000-859800, e (2) Costa Rica. IMBIO CR 1000276859.

O material foi observado macroscopicamente em um vídeo microscópio Olympus OVM 1000 NM com vídeo printer Sony UP 3000. Posteriormente os detalhes foram fotografados em um microscópio eletrônico de varredura (MEV) JEOL JSM-35 CF; os aumentos foram de 20X a 360X. O material foi previamente limpo com acetona e não se usou nem ultrassom nem metalização com o intuito de proteger o raro exemplar. As áreas estudadas foram: cabeça em vista dorsal e ventral, clípeo e anteclípeo, região óculo-ocelar, sinlipsis, bucula, genas, tubérculo antenífero, sulco estridulatório, escutelo, coxas do primeiro par de patas e articulação tibio-tarsal.

\section{RESULTADOS}

Cabeça com região ante-ocular 2,52 vezes mais longa que a pós-ocular, com toda a superfície, desde o clípeo até a região ocelar granulosa, com $1+1$ áreas mais lisas paralelas limitando a área central, clípeo retangular alargado na base, ultrapassado ligeiramente pelas genas.

Tubérculos anteníferos com uma projeção 

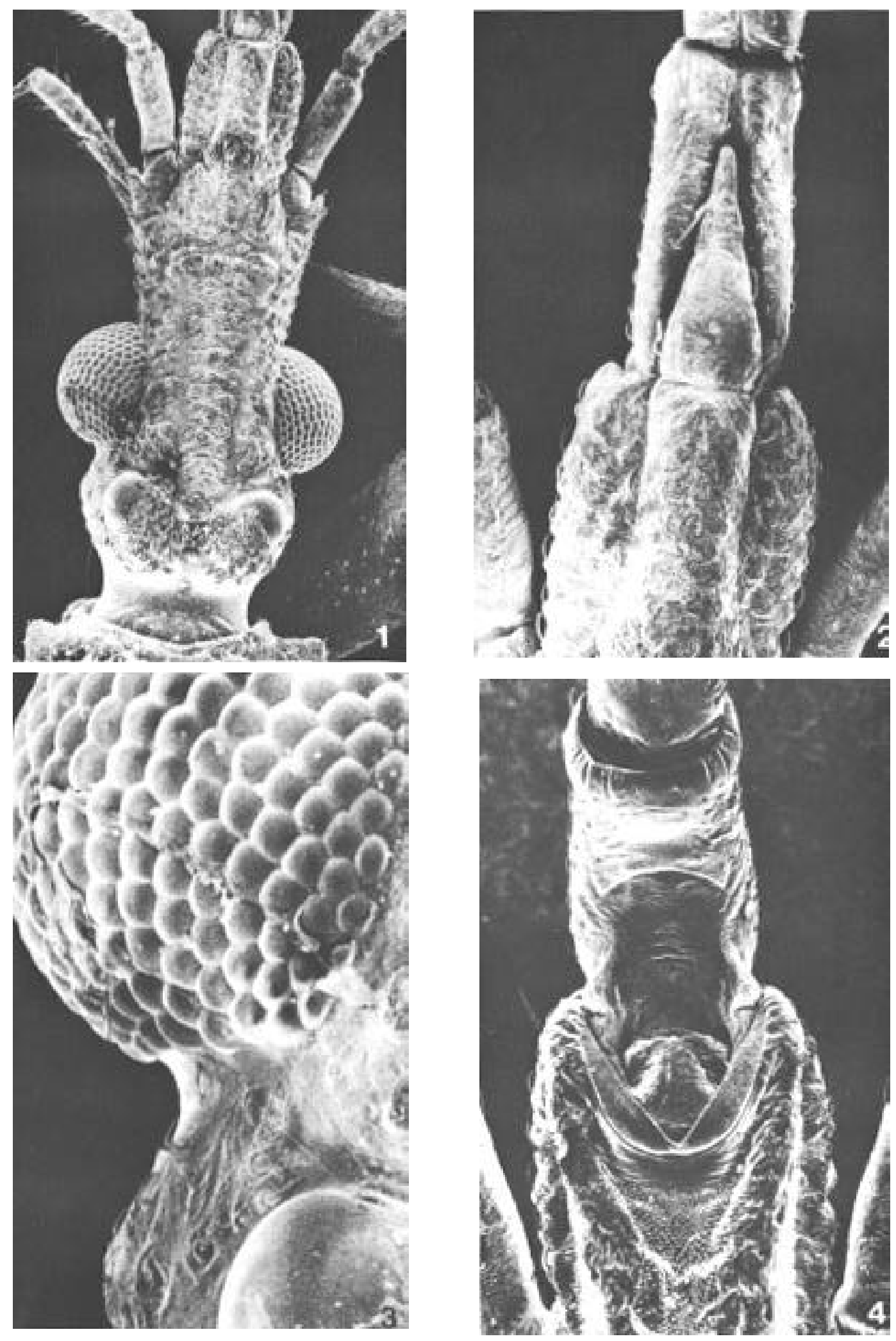

Triatoma ryckmani Zeledón \& Ponce, 1972. Detalhes em microscopia eletrônica de varredura. Fig. 1: cabeça vista dorsal em 33X. Fig. 2: genas, clípeo e labro 75X. Fig. 3: região óculo-ocelar 260X. Fig. 4: bucula, primeiro segmento do rostro, 150X. 

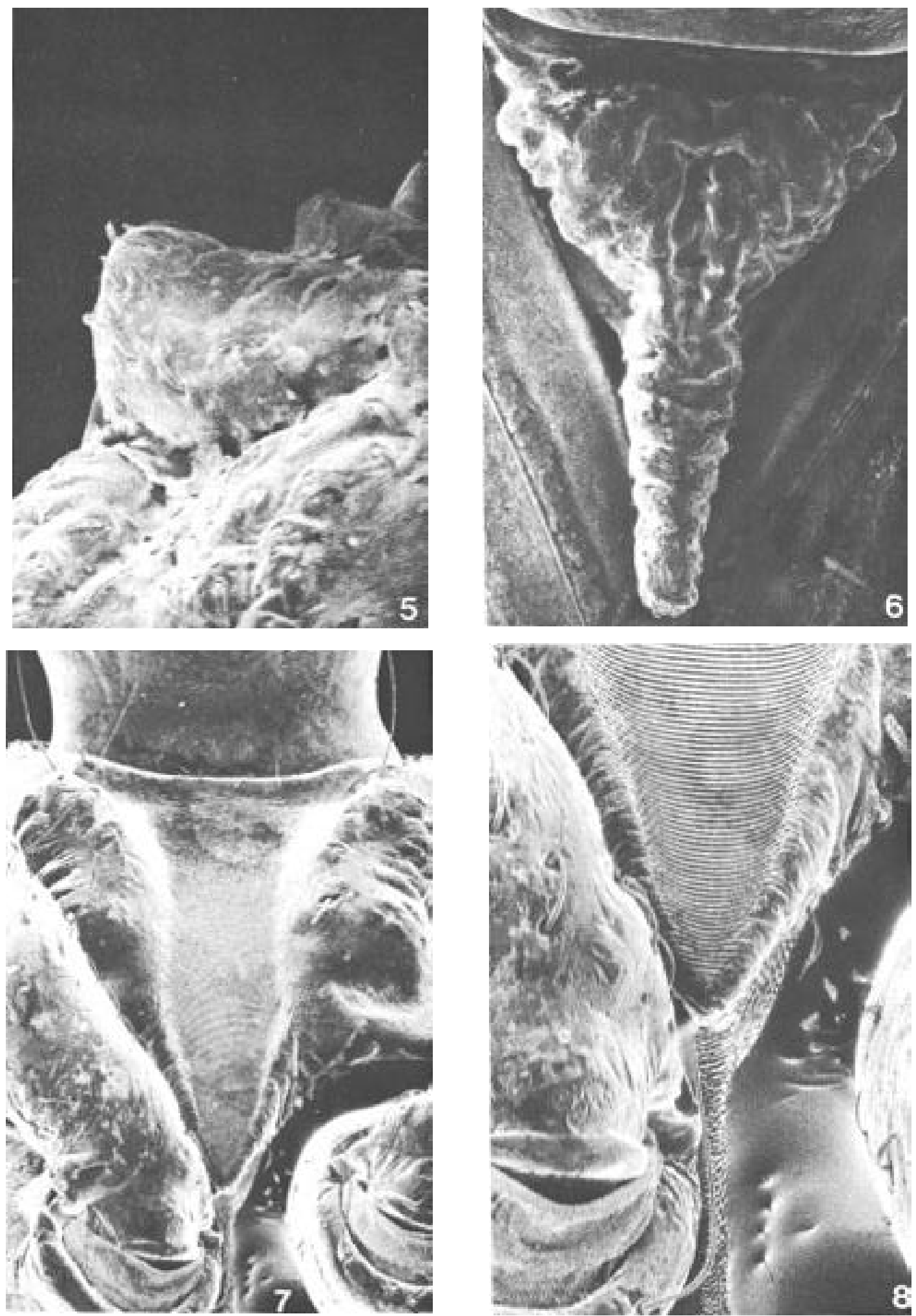

Triatoma ryckmani Zeledón \& Ponce, 1972. Detalhes em microscopia eletrônica de varredura. Fig. 5: ângulo antero-lateral no colar, 240X. Fig. 6: escutelo, 78X. Fig. 7: proesterno com o sulco estridulatório, 130X. Fig. 8: detalhe do extremo distal do sulco estridulatório, 300X. 
ápico-lateral saliente, olhos globosos com a margem anterior situada um pouco para trás do meio da cabeça, área pós-ocular bastante alargada, ocelos salientes, grandes, situados afastados do bordo posterior do olho, implantados em $1+1$ base saliente enfumada (Fig. 1).

Clípeo e genas de superfície granulosa e recoberta de pêlos curvos quase decumbentes, direcionados para os lados e para frente; labro com raros pêlos, glabro (Fig. 2).

Região óculo-ocelar com um sulco largo, deprimido, glabro com raros pêlos na região central e uma elevação de superfície irregular com algumas estrias na área externa próxima ao ócelo onde estão implantadas cerdas decumbentes que emergem de áreas deprimidas. Atrás do olho, contornando-o, existe um sulco que se conecta ao sulco inter óculo-ocelar (Fig. 3).

Rostro, entre o primeiro e segundo segmentos, estendido, observa-se uma área circular estriada. Abaixo apresenta uma área triangular com superfície uniformemente ponteada entre as buculas (Fig. 4).

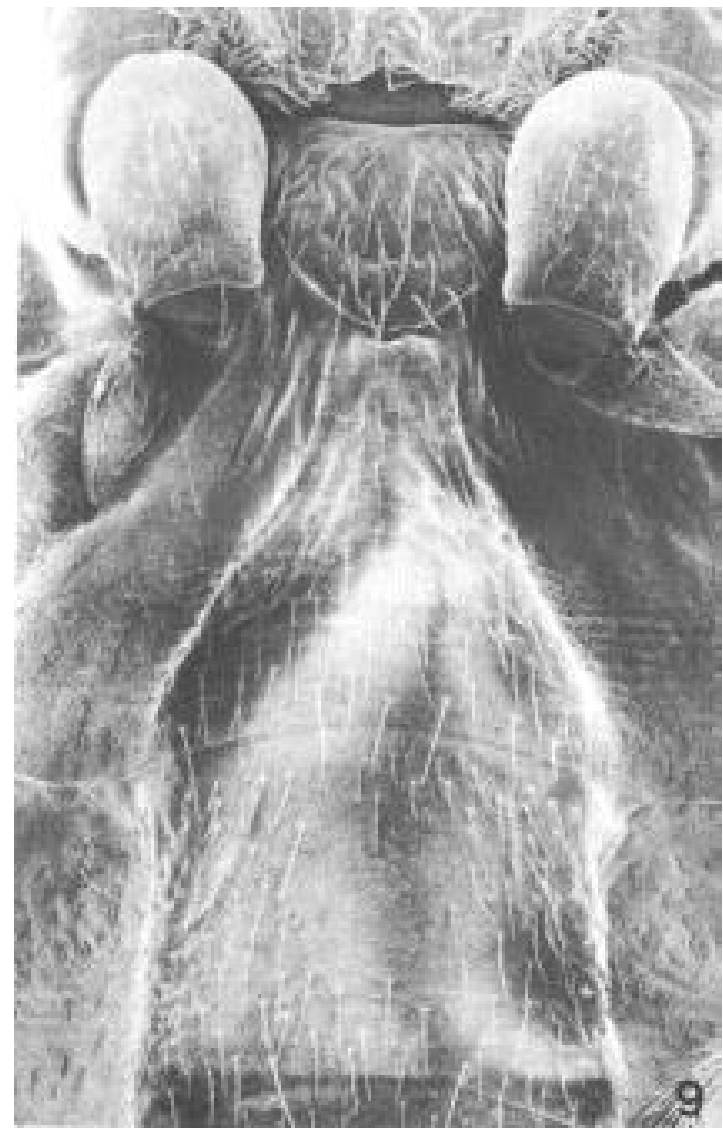

Ângulo anterolateral do pronoto com uma projeção na forma cônica, pouco elevada, com um sulco separando-a do lobo posterior do pronoto, com alguns pêlos fortes decumbentes nas áreas externas, enquanto que a área interna é de superfície irregular e glabra (Fig. 5).

Escutelo com duas áreas bem definidas, um corpo e ápice. O corpo possui uma projeção ondulada dirigida para trás em ambos os lados da linha mediana; os bordos do corpo são fortemente esculpidos e irregulares; na parte mediana existe um sulco estreito irregular e profundo que termina na base do ápice do escutelo que é 1.4 maior do que o corpo. $\mathrm{O}$ ápice do escutelo, cilíndrico, possui um sulco longitudinal, delgado, no quinto proximal que é uma prolongação existente no corpo; a parte cilíndrica possui esculturação transversal (Fig. 6).

Sulco estridulatório de formato triangular, base longa, bordos laterais elevados na parte anterior, superfície estriada com raros pêlos; o ápice do sulco possui uma abundante pilosidade (Fig. 7). Abaixo

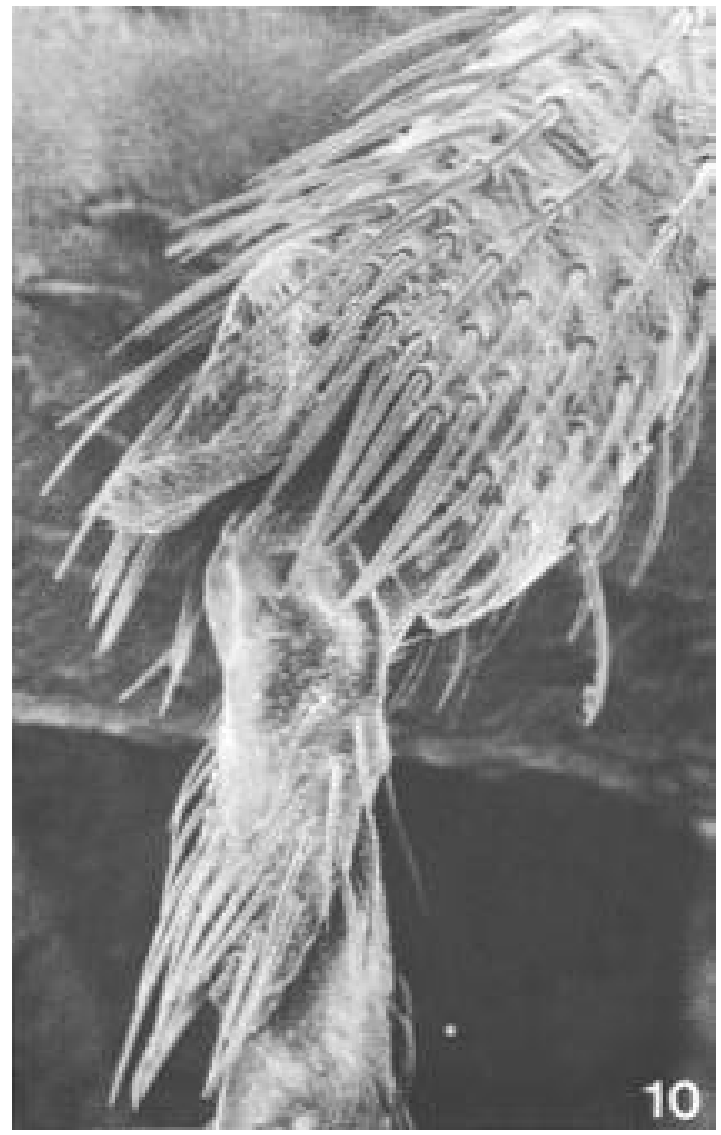

Triatoma ryckmani Zeledón \& Ponce, 1972. Detalhes em microscopia eletrônica de varredura. Fig. 9: metaesterno, base do abdomem, 48X. Fig. 10: ápice da tíbia e parte do tarso, fóssula esponjosa, 240X. 
do ápice do sulco estridulatório está localizada uma área estriada, granulosa, vermiforme prolongada.

Metaesterno cordiforme com a superfície lisa onde estão implantados numerosos pêlos longos. A região central e ventral do abdomem é deprimida e limitada por duas carenas paralelas; a área está recoberta de pêlos longos (Fig. 9).

Segundo par de patas apresenta uma fóssula esponjosa no extremo tibial, rodeado por pêlos longos e grossos (Fig. 10).

\section{DISCUSSÃO E CONCLUSÃO}

Este trabalho amplia o conhecimento morfológico desta espécie e cria ilações taxonômicas a respeito dos exemplares estudados. Os vários autores que usaram a técnica de MEV com fins taxonômicos (Lent \& Wygodzinsky 1979, Costa et al. 1991, Galindez et al. 1994, Carcavallo et al. 1994 a,b,c, 1995), dão ênfase às estruturas através dessa técnica. Baseado nesta abordagem T. ryckmani chama a atenção nas seguintes áreas: superfície dorsal da cabeça, especialmente na área do clípeo e genas, região óculo-ocelar, forma do pós-clípeo, forma e estrutura articular do ângulo antero-lateral do pronoto, forma e proporções do escutelo, aspecto do sulco estridulatório e formação vermiforme, superfície achatada ventral do abdomem, fóssula esponjosa e articulação tíbio tarsal.

Dois aspectos, a presença de fóssula esponjosa no segundo par de patas e o tipo de escutelo do exemplar examinado, criam interesse sob o ponto de vista taxonômico por não concordarem com as descrições de Zeledón e Ponce (1972), Lent e Wygodzinsky (1979), Sherlock e Morera (1988). Essas diferenças dos exemplares descritos e a maior semelhança com o alótipo da Costa Rica nos indicam que um maior número de exemplares deverá ser observado principalmente de populações autóctones de Honduras e Guatemala.

\section{REFERÊNCIAS}

Carcavallo RU, Jurberg J, Martinez A, Galindez Giron I 1994a. La región óculo-ocelar en el género Rhodnius Stal, 1859 (Hemiptera, Reduviidae, Triatominae). Entomol Vect 1: 21-32.

Carcavallo RU, Galindez I, Martinez A, Jurberg J 1994b. Cuticular structures of Triatominae. Their taxonomic significance. Entomol Vect 1: 79-91.

Carcavallo RU, Galindez I, Jurberg J, Martinez A 1994c. La región óculo-ocelar en el género Panstrongylus Berg, 1879 (Hemiptera, Reduviidae, Triatominae). Entomol Vect 1: 113-120.

Carcavallo RU, Galindez Giron I 1995. Systematic value of the scutellum in Triatominae (Hemiptera, Reduviidae). Entomol Vect 2: 23-29.

Costa JM, Jurberg J, Barth OM 1991. Estudos morfológicos de Cavernicola lenti Barrett \& Arias, 1985 (Hemiptera, Reduviidae, Triatominae). Mem Inst Oswaldo Cruz 86: 247-263.

Galindez I, Carcavallo RU, Valderrama A 1994. Erwinilas o cerdas interomatidiales en la subfamilia Triatominae. Entomol Vect 1: 93-96.

Lent H, Wygodzinsky P 1979. Revision of the Triatominae (Hemiptera, Reduviidae) and their significance as vectors of Chagas'disease. Bull American Mus Nat Hist 163: 125-520.

Sherlock I, Morera P 1988. Alótipo macho de Triatoma ryckmani Zeledón y Ponce, 1972. Rev Biol Trop 36: 423-428.

Zeledón R, Ponce C 1972. Descripción de una nueva especie de Triatoma de Honduras, América Central (Hemiptera: Reduviidae). Rev Biol Trop 20: 275279. 
732 Morfologia Externa de T. ryckmani - RU Carcavallo et al. 